\title{
LES MARQUES RÉGION À L'ÉPREUVE DE LA RÉFORME TERRITORIALE
}

\author{
Corinne Rochette, Cédrine Zumbo-Lebrument, Pierre Féniès
}

Association de recherches et publications en management | « Gestion 2000 »

2015/6 Volume 32 | pages 55 à 72

ISSN 0773-0543

Article disponible en ligne à l'adresse :

http://www.cairn.info/revue-gestion-2000-2015-6-page-55.htm

\section{Pour citer cet article :}

Corinne Rochette et al., "Les marques région à l'épreuve de la réforme territoriale », Gestion 2000 2015/6 (Volume 32), p. 55-72.

Distribution électronique Cairn.info pour Association de recherches et publications en management.

(C) Association de recherches et publications en management. Tous droits réservés pour tous pays.

La reproduction ou représentation de cet article, notamment par photocopie, n'est autorisée que dans les limites des conditions générales d'utilisation du site ou, le cas échéant, des conditions générales de la licence souscrite par votre établissement. Toute autre reproduction ou représentation, en tout ou partie, sous quelque forme et de quelque manière que ce soit, est interdite sauf accord préalable et écrit de l'éditeur, en dehors des cas prévus par la législation en vigueur en France. Il est précisé que son stockage dans une base de données est également interdit. 


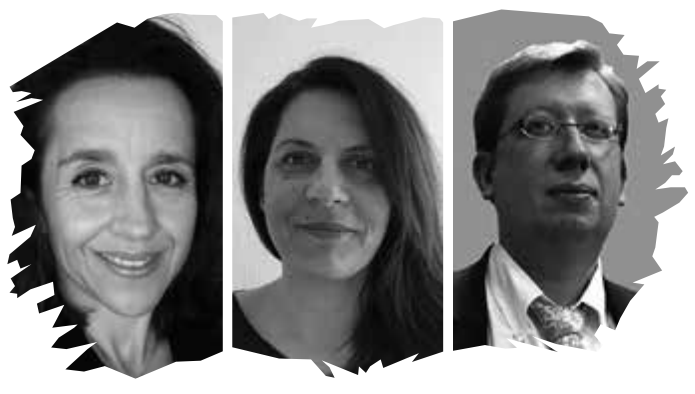

Corinne ROCHETTE

Maitre de conférences, Ecole Universitaire de Management - IAE d'Auvergne

Cédrine ZUMBO-LEBRUMENT

Doctorante CIFRE, Agence Régionale de Développement Economique d'Auvergne (ARDE), Université Paris Ouest Nanterre la Défense ; CEROS

\section{Les marques région à l'épreuve de la réforme territoriale}

e place branding constitue un véritable phénomène : depuis 5 ans, 50 marques Lterritoriales ont été créées'. D'abord utilisé par les villes et les pays au début des années 2000, ce phénomène de marquage se diffuse en France sur une nouvelle échelle territoriale : les régions. Après la Bretagne, l'Auvergne, l'Alsace, d'autres régions viennent, très récemment, d'amorcer cette démarche de marketing territorial alors même que le gouvernement annonçait en janvier 2014 un projet de réforme territoriale. La nouvelle organisation territoriale de la république ("NOTRe ") constitue un projet politique de rationalisation de la gestion publique fondé sur la réduction du nombre de régions de 22 à 13. Certaines régions ont engagé des ressources importantes dans la construction d'une marque. Elles sont parvenues à mobiliser les parties prenantes autour d'un projet de territoire que la réforme territoriale vient réinterroger.

$\mathrm{Si}$ le regroupement, tout comme la scission de territoires n'est pas une chose nouvelle, ces manifestations de la souveraineté du politique conduisent fréquemment à des débats sur l'identité. Ces modifications du périmètre administratif sont vécues de manière contrastée. Dans certains cas, elles constituent une évolution naturelle pour réunifier des territoires à l'identité

\footnotetext{
1 Source ADETEM
}

similaire $^{2}$ dans d'autres cas elles sont vécues comme un mariage forcé, les « mariés " pouvant craindre de perdre leur singularité et de voir leur identité altérée. Le débat autour du choix du nom de ces nouveaux ensembles restitue cette volonté de défendre une identité qui s'exprime dans la marque. Le choix d'un nom n'est pas neutre, il implique un dialogue, des délibérations,

2 RDA et RFA pour former l'Allemagne, l'actuel débat sur la fusion des départements de Savoie et de Haute Savoie, ou Bas-Rhin et Haut-Rhin illustre ce point. 
une conversation. Le changement de nom exprime à la fois une transformation et une forme d'autorité qui conduit à repenser la place des marques actuelles et à s'interroger sur leur avenir.

En nous appuyant sur les recherches en gestion portant sur le changement de nom de marque et les conséquences des fusions en matière de gestion des marques ainsi que sur une typologie des stratégies de marquage nous proposerons des scénarios d'évolution possible de la marque région. Ces scénarios sont ensuite confrontés à l'étude d'un cas particulier, celui de la marque régionale Auvergne Nouveau Monde. Les résultats d'une enquête destinée à identifier la perception que les acteurs socio-économiques ont de la marque Auvergne Nouveau Monde permettent d'alimenter la discussion sur l'avenir de cette marque et de dégager les pistes probables de son évolution.

\section{Quand la réforme} territoriale questionne le changement de nom?

\section{Les enjeux du changement de nom}

Dans le domaine de la gestion d'entreprises nombreuses sont les sociétés qui modifient leur nom ou celui de leurs marques tant dans une perspective de réduction du nombre de marques que d'internationalisation de celles-ci (Collanges, 2005). Le nom est essentiel au point que sa modification est considérée comme un évènement majeur (Barnejee, 2008). Ce changement représente un risque particulier pour l'entreprise. II peut modifier l'attitude du consommateur et induire des changements cognitif (perte de repère), conatif (réduction de la fréquentation et des achats) et affectif (distension du lien).

Bien que le nom et la marque soient généralement traités conjointement, certains chercheurs distinguent nettement ces deux notions (Collanges, 2005 ; Laurent, 2006). Muzellec (2006) opère une distinction entre le nom d'entreprise et la marque. Selon lui, le nom concerne l'identité de l'entreprise, l'expression de la culture interne, il doit être accepté par un large public et sa communication est secondaire. Il forme les identités des membres (Lipiansky, 1993), développe la culture (Roy, 1960) et contribue à maintenir les relations de pouvoir (Banks, 1990). La marque, elle, se réfère plus à la capacité à attirer l'attention, elle cible le client. Elle apparait clairement comme un instrument en lien avec le marché. Destinée à séduire, elle porte en elle une promesse, elle valorise une offre. Ces approches disjointes renvoyant à l'internalisation pour le nom et à une certaine externalisation pour la marque sont traitées simultanément par Kapferer (1988) dans son prisme 
d'identité qui illustre la complémentarité du nom et de la marque (FlorenceValette et Barnier, 2011).

Le changement de nom de marque a été très étudié (Merrilees et Miller, 2008 ; Muzellec et Lambkin, 2006) que la marque soit corporate, de produits ou de service, tout comme sa création (Klink, 2003), sa conception (Kapferer, 1991, 2002).

Cegarra (1994) met en avant le rôle déterminant de l'identité nominale dans le choix d'une stratégie de marquage. II identifie deux options soustendant le changement de nom.

- L'une consiste en la restructuration de l'identité nominale soit par réduction du nombre de marques porté par le produit (simplification) soit par adjonction d'une marque à celle existante afin de préciser le positionnement du produit (cautionnement).

- L'autre se réfère à la modification de I'identité nominale (substitution), elle consiste à abandonner une marque pour une autre.

Le choix de l'une ou l'autre de ces options repose sur des logiques stratégiques, mais aussi politiques dans la mesure où le choix d'un nom (ici d'une marque) exprime une forme de pouvoir.

Le choix raisonné d'une identité nominale nécessite de se pencher sur plu- sieurs variables : le statut de la marque et sa notoriété préalable, son positionnement, les catégories de produits couvertes (Aimé-Garnier et Lai, 2008) car le changement de nom d'un produit modifie l'attitude des consommateurs (Collange, 2005) que la marque adoptée soit ancienne ou nouvelle. Cinq situations influencent l'attitude et doivent être étudiées (Collange, 2005) : la similarité entre l'ancienne et la nouvelle marque, la cohérence entre la nouvelle marque et le produit, la différence de notoriété et $d$ 'image entre les deux marques ainsi que l'attachement à l'ancienne marque. Le choix de la nouvelle marque est guidée par la congruence perceptuelle (Aaker et Keller 1990), afin que le consommateur (dans notre cas l'usager du territoire) perçoive l'association entre les deux marques comme logique et compatible. Ces approches permettent d'identifier la marque la mieux adaptée. Le changement de nom remplit aussi une fonction $\mathrm{d}^{\prime}$ annonce, de transformation (Stuart et Muzellec, 2004 ; Boistel, 2012).

Boistel (2012) montre que la modification du nom d'entreprise est bien un signal donné au marché que quelque chose a changé au sein de l'entreprise (Stuart et Muzellec, 2004). Cette modification doit intervenir postérieurement aux transformations importantes subies par l'organisation. Le changement de nom a pour objectif de créer une nouvelle image positive autour des valeurs de la nouvelle entité. II renvoie à une 
logique ambivalente. Il est à la fois perçu comme stratégique car il exprime un nouveau départ pour l'organisation, avec la chance de créer une nouvelle image positive (Stuart et Muzellec, 2004), mais parallèlement il génère des coûts de nature marketing (refonte des supports de communication, perte de repère pour le client) (Kapferer, 1994 ; Keller, 2002). S'il n'est pas associé à une modification profonde de l'organisation (Stuart et Keller, 2004), il peut constituer une signalisation prématurée (Dowling, 1996) ${ }^{3}$ et être un facteur d'échec.

Le changement de nom peutêtre analysé comme le souhait de développer une image et une réputation favorables en envoyant des signaux positifs vers l'ensemble des parties prenantes.

\section{Des leçons à tirer des fusions d'entreprises}

La réforme territoriale s'apparente à une fusion d'entités (administratives), mais aussi potentiellement de marques si les entités administratives qui fusionnent disposent de leur(s) propre(s) marque(s). Dans le cas de la nouvelle région "Auvergne - Rhône-Alpes » une des entités porte une marque région (Auvergne Nouveau Monde) et l'autre une marque ville forte (Only Lyon pour la région Rhône-Alpes). Aussi, il peut

3 Downling parle de piège, "corporate identity traps» être fait un parallèle entre cette situation et celle rencontrée dans les entreprises.

Les travaux sur les alliances et les fusions d'entreprises (corporate et produit) fournissent un cadre de réflexion utile. Ils permettent de dégager deux grandes options : l'une se caractérise par la disparition des identités (marque corporate) qui fusionnent (ou seulement celle de la firme achetée), I'autre par leur maintien. Ce deuxième cas, celui du maintien, est généralement lié à la présence d'une marque forte de la firme achetée qu'il convient de conserver tout en cherchant une certaine mutualisation des moyens. L'adoption de l'une ou l'autre de ces deux options n'est pas nécessairement très tranchée. En effet, elle peut donner lieu à des ajustements, des corrections qui s'expriment parfois sur une échelle de temps longue. Le cas de Peugeot et Citroën est une bonne illustration de ce phénomène. Rigaud (2009) dans l'analyse de ce cas de fusion (intervenue en 1976) montre le caractère interminable de la fusion. II met ainsi en évidence l'existence d'un mouvement entre autonomie et mutualisation. Si immédiatement après la fusion, dans une première phase la mutualisation est recherchée (réduction des coûts, rationalisation des processus), celle-ci fait apparaitre des effets négatifs, dont l'atténuation de la marque pouvant conduire à sa disparition, s'enclenche alors une deu- 
xième phase, celle d'autonomisation des marques. Celle-ci se traduit par une mise en concurrence des marques considérée comme saine et stimulante. Mais cette deuxième phase trouve elle aussi ces limites et un retour vers la mutualisation se produit et ainsi de suite. Ce mouvement de balancier illustre la délicate articulation entre mutualisation et autonomie des processus de gestion des marques qui peut être considéré comme un tâtonnement vers une pratique équilibrée.

\section{Le positionnement de la recherche}

Ces travaux mettent en avant les limites et difficultés à utiliser les grilles de lecture des marques commerciales ou corporate sur les marques région en raison de leurs singularités. En effet, ce sont des marques co-construites et/ou partagées, portant sur un objet particulier qui est le territoire, dont finalité n'est pas prioritairement économique et qui comportent des enjeux politiques forts.

\section{Un état des lieux des mar- ques territoire}

Les territoires qui sont amenés à fusionner dans le cadre de la réforme territoriale présentent $d$ 'importantes différences en matière de stratégie de marquage. Certaines régions ont développé des marques régionales, d'autres pas, mais ces dernières peuvent néanmoins être porteuses parfois de marques se situant sur d'autres échelons territoriaux (départements, pays de tradition, villes). La différence est donc nette avec les entreprises qui, elles, disposent d'une marque corporate et de marques produits et services savamment articulées. Sur un territoire donné la coexistence de marques se situant à divers échelons territoriaux est délicate, leur mise en cohérence autour d'un projet de territoire inclusif se traduit par une mise en tension des différents niveaux de marquage et I'affirmation de la dimension politique (Chanut et Rochette, 2012 ; Rochette, 2012; Houllier-Guibert, 2012) : les villes, départements voire les pays de tradition tentant eux aussi d'exister.

Dans le cas qui nous intéresse, celui de la fusion des régions Auvergne et Rhône-Alpes, à l'échelle "région » seule la région Auvergne dispose d'une marque, pour la région Rhône-Alpes c'est la capitale régionale qui a fait l'objet d'une démarche de marketing territorial poussée avec la marque ville Only Lyon. Ainsi, le développement d'une stratégie homogénéisée de gestion des marques portées par le nouveau territoire Auvergne RhôneAlpes (dont l'appellation administrative est actuellement discutée) relèverait plus de l'alliance entre marques que de la fusion. 
Tableau 1 : Stratégies de marquage, champs d'action et dénominations retenus

\begin{tabular}{|c|c|c|}
\hline & Champ d'action global & Champ d'action plus ciblé \\
\hline $\begin{array}{l}\text { Choix d'une même appellation } \\
\text { territoire administratif et marque }\end{array}$ & « Limousin » & $\begin{array}{l}\text { «Bretagne » (économie) } \\
\text { «Alsace » (économie) }\end{array}$ \\
\hline $\begin{array}{l}\text { Choix d'une appellation diffé- } \\
\text { rente territoire administratif et } \\
\text { marque }\end{array}$ & $\begin{array}{l}\text { "Auvergne Nouveau } \\
\text { Monde» }\end{array}$ & $\begin{array}{l}\text { "Sud-Ouest France » } \\
\text { (agriculture et filières agroalimentaires } \\
\text { pour les régions Aquitaine et Midi- } \\
\text { Pyrénées) } \\
\text { "Sud-de-France " } \\
\text { (filières agroalimentaires, viticoles et } \\
\text { tourisme pour la région Languedoc } \\
\text { Roussillon) }\end{array}$ \\
\hline
\end{tabular}

Source : Les auteurs

La distinction nom et marque est à considérer avec attention pour mieux appréhender le marketing territorial, son contexte et les dynamiques en jeu. Si le nom renvoie à l'appellation d'une structure administrative (conseil régional) territorialisé (Auvergne), la marque relève de la façon dont on entend être perçu par les cibles (citoyens, touristes, étudiants, entreprises, ...) et se positionner face aux territoires concurrents (Anhold, 2006). Elle est l'expression d'un projet et d'une ambition. Une revue des stratégies de marques déployées par les régions permet de mettre en évidence quatre situations (tableau 1) selon que le nom de la marque se distingue de ce celui de l'entité administrative et selon le champ d'utilisation (marque globale, ou marque ciblée).

En ce qui concerne le champ d'action, certaines marques adoptent une approche globale. Elles concernent tous les domaines (touristique, économique, culturel, de formation) tel est le cas pour l'Auvergne et le Limousin, $d^{\prime}$ autres se recentrent sur un univers d'action plus restreint (le tourisme, l'économie, l'agriculture et l'agroalimentaire). Quant à la dénomination retenue, deux grandes orientations peuvent être dégagées. La première consiste à utiliser le même terme pour désigner le territoire et la marque (I'Alsace, la Bretagne, le Limousin), la seconde consiste à développer une marque portant une appellation différente de celle désignant le territoire administratif, c'est le cas de la marque «Auvergne Nouveau Monde ».

Le regroupement régional est de manière évidente porteur $d$ 'un déficit identitaire, la question de la dénomination constitue le point central d'un modèle identitaire dont la marque est l'expression (Cardy, 1997). Dans ce contexte quelle est la place des marques pour 
construire l'identité de la nouvelle région? Les marques actuelles peuventelles être maintenues, redéployées ? Leur champ d'action doit-il être redéfini ?

\section{Les grands scenarios envisageables}

La réforme territoriale nécessite de repenser en profondeur la marque région. Partant du principe que la nouvelle marque est le signal que quelque chose a changé (Stuart et Muzellec, 2004 ; Boistel, 2012), la transformation de la structure administrative du territoire offre une opportunité pour créer une image positive et communiquer sur les ambitions et les enjeux associés aux nouvelles régions et envoyer des signaux positifs aux acteurs les plus en attente d'évolutions (entreprises, porteurs de projets, étudiants, habitants). Elle est l'opportunité de transformer l'identité organisationnelle (Lejeune et Vaz, 2011), de donner une existence au nouveau territoire social et politique. En nous fondant sur les travaux réalisés sur les marques commerciales (corporate et produits) nous proposons trois scénarios (qui constituent des propositions de configurations des identités nominales régionales).

- Un premier scénario consiste en changeant de nom à créer une nouvelle marque (modification nominale) par substitution (Cegar- ra, 1994) aux actuelles marques régions existantes. Ce scénario offre l'avantage de limiter les tensions entre les entités regroupées en adoptant une appellation ménageant les susceptibilités et en reconstruisant une nouvelle identité globale tout en dégageant un message fort. Elle doit être le marqueur d'un projet de territoire rassembleur. Une déclinaison de ce scénario peut être de réutiliser dans la nouvelle identité nominale des repères nominaux $d^{\prime}$ 'une ou de plusieurs des actuelles marques ${ }^{4}$ soit parce que les termes utilisés sont porteurs et s'appliquent bien aux projets du nouvel ensemble lexemple « Nouveau Monde » pour Auvergne Rhône-Alpes, ou « innov » pour l'ensemble « Champagne, Ardennes, Lorraine, Alsace ») soit en raison du capital crée par l'actuelle marque (notoriété, image positive). Il consiste aussi à penser la nouvelle marque dans des applications plus sectorielles (culture, formation...).

- Un deuxième scénario repose sur la restructuration de l'identité nominale en adjoignant aux actuelles marques une nouvelle marque. L'objectif est de donner une existence au nouvel ensemble tout en conservant les marques actuelles. Elle constitue une stratégie de co-

\footnotetext{
4 Précisons qu'il n'y a pas de cas pour lequel les régions issues de la réforme territoriale concernent deux ou plusieurs régions ayant développées des marques territoires globales.
} 
marquage (Cegarra et Michel 2001 ; Michel, 2004). Cette option se traduit d'une part par des surcoûts liés à l'entretien de plusieurs marques de portée équivalente et aux cibles similaires, ce qui va à l'encontre de la volonté de rationalisation et de mutualisation des moyens, et d'autre part peut conduire à un brouillage du message (cf. Rigaud, 2009 sur le cas de Peugeot et Renault). Ce scénario parait difficilement envisageable pour des marques dont l'identité nominale est très institutionnelle et le champ couvert global, comme c'est le cas pour la marque "Limousin», cependant il ne peut être écarté en particulier pour des territoires où les résistances au regroupement sont fortes. II ne serait dans ce cas probablement qu'un scénario de transition. Pour les marques reprenant comme appellation celle de la région administrative ("Bretagne », "Alsace »), mais adoptant une action ciblée, une adaptation nominale serait probablement nécessaire (par exemple «Bretagne-business») pour une action sur un périmètre plus restreint.

- Un troisième scénario se trouve dans la possibilité de conserver les marques existantes dont le champ $d$ 'action est ciblé et de redéfinir le périmètre d'action sectoriel de celles actuelles plus globales (comme "Auvergne Nouveau Monde ») afin de dégager des objectifs et des cibles plus ajustés. II s'agit de restreindre leur périmètre actuel pour plus d'efficacité et une concentration des moyens sur des objectifs précis, de permettre la coexistence avec la signature institutionnelle voire avec une marque plus administrative de la nouvelle région.

L'adoption de l'un ou l'autre de ces scénarios repose d'abord sur son acceptation par les parties prenantes, c'est-à-dire celles qui font la marque et lui donnent une existence, aussi notre démarche consiste à interroger les parties prenantes de la marque région "Auvergne Nouveau Monde » afin d'identifier le scénario qui pourrait être accepté et donc la place qui pourrait être faite à cette marque.

La marque «Auvergne Nouveau Monde» a été créée en septembre 2011 à l'initiative du Conseil Régional d'Auvergne et de ses quatre agences de développement territoriales rejoints par de grandes entreprises régionales, des PME, des festivals, des lieux culturels, des associations, des universités, etc., pour atteindre en 2014, environ 700 adhérents à l'association chargée de développer la marque. Portée sous forme associative, cette marque repose sur une volonté politique d'instaurer une démarche de marque «partagée» entre habitants, collectivités, institutionnels, entreprises, et universités - fondée sur une approche collaborative (coconstruite). 


\section{L'étude empirique}

\section{Méthodologie}

Pour interroger le devenir de la marque partagée Auvergne Nouveau Monde (ANM), nous avons recueilli la perception des acteurs socio-économiques auvergnats qui partagent pour certains la marque, à partir d'une enquête réalisée en juillet 2014. Le questionnaire a été construit sur la base des orientations données par le délégué général de l'association ANM à partir des thématiques dégagées lors des réunions avec les membres du bureau de l'association. Le questionnaire était structuré autour de trois thèmes centraux: l'attractivité de la région Auvergne, la fusion avec la région Rhône-Alpes et la marque Auvergne Nouveau Monde. Il a été envoyé par mail, à l'initiative de l'association Auvergne Nouveau Monde, aux adhérents de la chambre de commerce et $d^{\prime}$ 'industrie d'Auvergne et dépouillé sous le logiciel Sphinx. Les réponses constituent des éléments descriptifs de la manière dont est perçue
l'Auvergne (en termes d'attractivité, de notoriété et d'image), elles rendent compte de la place de la marque et de son action ainsi que des conséquences perçues de la fusion sur la marque. Elles fournissent des indices pour estimer si pour les interrogés cette fusion peut constituer la base d'un nouveau départ ou si elle doit s'inscrire dans la continuité.

Les 227 questionnaires retournés complétés restituent les grandes catégories d'acteurs impliquées dans les démarches de marquage territorial (tableau 2) : les entreprises, les associations, les collectivités et institutions (organisations consulaires) ainsi que les organismes de formation supérieure (université, écoles). Près de $54 \%$ des répondants sont des entreprises avec une cette surreprésentation des entreprises de moins de 50 salariés (76\% d'entre-elles), très caractéristique du tissu d'entreprises auvergnates. ${ }^{5}$

5 Sur les 227 répondants 9 n'ont pas rempli la rubrique "type d'organisation", mais ont répondu aux questions.

Tableau 2 : Structure des répondants (Juillet 2014)

\begin{tabular}{|l|c|c|}
\cline { 2 - 3 } \multicolumn{1}{c|}{} & Effectifs & $\%$ Rep. \\
\hline Une entreprise & 116 & $53,2 \%$ \\
\hline Une association & 56 & $25,7 \%$ \\
\hline Une collectivité ou une autre institution & 35 & $16,1 \%$ \\
\hline Une universtité, école, ... & 11 & $5 \%$ \\
\hline Total & $\mathbf{2 1 8}$ & $\mathbf{1 0 0} \%$ \\
\hline
\end{tabular}




\section{Principaux résultats et commentaires}

L'enquête permet de mettre en lumière des éléments qui peuvent orienter le choix d'une stratégie de marquage (scénario) plutôt qu'une autre. Si l'Auvergne dispose de facteurs d'attractivité, elle souffre d'un relatif déficit de notoriété et d'image que la stratégie déployée par la marque ANM n'a pas permis de suffisamment combler.

\section{La perception de l'attractivité}

L'objectif premier des marques région est de contribuer à renforcer l'attractivité de leur territoire et sa lisibilité. Une enquête IPSOS réalisée auprès de 1500 personnes habitant hors Auvergne en $2012^{6}$ lors du lancement de la marque ANM avait permis de mettre en évidence, les éléments de I'attractivité de l'Auvergne : le tourisme et les atouts environnementaux (nature, situation géographique). A ce momentlà, seuls $18 \%$ des français pensaient que l'Auvergne était attractive pour $s^{\prime} y$ installer. La perception d'un niveau d'équipement insuffisant, d'un déficit

6 Une étude IPSOS réalisée en 2012 sur un échantillon de 1500 personnes habitant en France hors Auvergne met en évidence un décalage entre l'image que les non auvergnats ont de l'Auvergne et la réalité. Pour plus de détails voir : http://www.auvergnenouveau-monde.fr/sites/default/files/users/572/ files/Synth\%C3\%A8se\%20Enqu\%C3\%AAte\%20 I psos $\% 20$ Auvergne $\% 20$ Nouveau $\% 20$ Monde\%20DEF.pdf de dynamisme culturel et surtout économique contribuaient à la classer en $14^{\mathrm{ème}}$ position des régions françaises. Les résultats de notre enquête (juillet 2014) corroborent dans les grandes lignes ceux de l'enquête IPSOS, quelques différences. La perception de l'attractivité s'inscrit dans une dynamique globalement positive (tableau 3) mais les scores sur les composantes de l'attractivité sont très contrastés.

$73,6 \%$ (167) des acteurs économiques sondés jugent positivement l'attractivité la région, $26,4 \%(60)$ l'estiment négative. La progression s'explique principalement par l'évolution des attractivités touristique $(87,2 \%)$ et universitaire $(72,3 \%)$ qui obtiennent des scores élevés. Celles démographique et économique contribuent plus faiblement à ce progrès. Ceci peut s'expliquer par la mise en place d'actions ciblées sur des secteurs qui constituent naturellement une ressource et un atout pour le territoire (l'environnement) ou qui sont considérés comme une priorité pour l'avenir (la jeunesse pour l'offre universitaire).

Si le périmètre d'action voulue pour la marque ANM est global, il semblerait que ces actions profitent plus à certains domaines, ce qui pourrait, pour l'avenir, conduire assez naturellement la marque à resserrer son périmètre d'action.

La fusion de l'Auvergne avec la $2^{\text {ème }}$ région française (Rhône-Alpes) n'aurait 


\section{Tableau 3 : L'attractivité de l'Auvergne (Juillet 2014)}

Au regard de cette définition, pensez-vous que l'Auvergne a amélioré ces performances d'attractivité ces 10 dernières années d'un point de vue:

\begin{tabular}{|l|c|c|c|c|c|c|}
\cline { 2 - 7 } \multicolumn{1}{c|}{} & \multicolumn{2}{c|}{ Oui } & \multicolumn{2}{c|}{ Non } & \multicolumn{2}{c|}{ Total } \\
\cline { 2 - 7 } \multicolumn{1}{c|}{} & Eff & $\%$ Rep. & Eff & $\%$ Rep. & Eff & $\%$ Rep. \\
\hline Démographique & 115 & $55,8 \%$ & 91 & $44,2 \%$ & 206 & $100 \%$ \\
\hline Economique & 122 & $58,9 \%$ & 85 & $41,1 \%$ & 207 & $100 \%$ \\
\hline Touristique & 191 & $87,2 \%$ & 28 & $12,8 \%$ & 219 & $100 \%$ \\
\hline Universitaire & 149 & $72,3 \%$ & 57 & $27,7 \%$ & 206 & $100 \%$ \\
\hline Général & 153 & $73,6 \%$ & 55 & $26,4 \%$ & 208 & $100 \%$ \\
\hline Total & 730 & $69,8 \%$ & 316 & $30,2 \%$ & 1046 & $100 \%$ \\
\hline
\end{tabular}

qu'un effet limité sur l'amélioration de l'attractivité de l'Auvergne : 44\% (100) des répondants pensent que cela améliorera l'attractivité, mais pour 22\% (50) aucune amélioration n'est à attendre. Ceci semble conforter l'idée que le renforcement de l'attractivité dans l'esprit des répondants est plus une question de communication volontaire et ciblée que d'affichage institutionnel (une même grande région), faisant passer au second plan les actions et politiques publiques qui constituent pourtant les instruments de I'attractivité d'un territoire. La méconnaissance en juillet 2014 du projet de territoire pour la nouvelle région explique probablement, en partie ces chiffres. Par ailleurs, les tris croisant la perception de l'attractivité de l'Auvergne avec l'origine géographique des répondants mettent en lumière une perception contrastée : les répondants du département du Cantal perçoivent une attractivité beaucoup plus faible $(66,7 \%)$ alors que pour $90 \%$ des répondants de I'Allier, I'Auvergne est attractive. Pour les deux autres départements Haute-Loire $(78,3 \%)$ et Puy-deDôme les $(71,7 \%)$ les chiffres sont très proches. Les tris croisant le profil du répondant (entreprises, associations, collectivités, universités/écoles) et la perception de l'attractivité permettent de constater que les entreprises et universités (respectivement $66,4 \%$ et $63,6 \%$ jugent la région moins attractive que les associations $(83,9 \%)$ et les collectivités (80\%).

L'image de l'Auvergne et la place de la marque ANM

La marque en tant qu'outil de communication et de promotion ne remplit pas pleinement son office: près de $54 \%$ des répondants jugent la promotion peu satisfaisante ou insatisfai- 


\section{Tableau 4 : Evaluation de la promotion de l'Auvergne (Juillet 2014)}

51. Comment jugez-vous la promotion globale de l'Auvergne aujourd'hui?

\begin{tabular}{|l|r|r|}
\cline { 2 - 3 } \multicolumn{1}{c|}{} & Effectifs & $\%$ Obs. \\
\hline Très satisfaisante & 3 & $1,3 \%$ \\
\hline Satisfaisante & 99 & $43,6 \%$ \\
\hline Peu satisfaisante & 110 & $48,5 \%$ \\
\hline Insatisfaisante & 12 & $5,3 \%$ \\
\hline Sans opinion & 3 & $1,3 \%$ \\
\hline Total & $\mathbf{2 2 7}$ & $\mathbf{1 0 0} \%$ \\
\hline
\end{tabular}

sante (tableau 4), probablement en raison d'une difficulté à faire émerger un réel positionnement partagé. Le positionnement constitue un préalable déterminant de la stratégie de marque (Cegarra, 1994, Aitken \& Campelo, 2011 ; Moilanen \& Rainisto, 2008 ; Aimé-Garnier, 2008), la nouvelle organisation territoriale est l'occasion de repenser le positionnement afin de pouvoir construire une véritable image qui fait aujourd'hui défaut.

En effet, pour moins de $50 \%$ des répondants l'image de l'Auvergne à l'extérieur' est positive (très bonne pour $1,3 \%$ et bonne pour $47,6 \%$ ). $24,2 \%$ l'estiment mauvaise et très mauvaise mais surtout pour $26,4 \%$ l'Auvergne n'a pas d'image. L'explication de ce déficit d'image perçue et

7 Celle-ci est appréhendée à partir de ce que le répondant pense de la façon dont on parle de l'Auvergne. absence d'image va de pair avec une notoriété insuffisante de la région. Pour 45,5\% (92) des répondants, I'Auvergne est connue, mais pour $26,9 \%$ elle ne l'est pas. Ce score est amplifié par le fait que $31,7 \%$ des répondants «non-auvergnats » ont une difficulté à situer géographiquement la région et à la qualifier. La fusion avec une région économiquement puissante peut avoir un effet positif sur la notoriété et l'image. L'avis des répondants sur ce point est divergent (tableau 5). Si pour $44,6 \%$ la notoriété pourrait $s$ 'en trouver améliorée et pour $41,5 \%$ l'image pourrait progresser, ils sont près de $37 \%$ à penser que ni l'image ni la notoriété de l'Auvergne bénéficieront de ce rapprochement. $20 \%$ des répondants ne se positionnent pas sur les effets de la fusion, un chiffre révélateur d'une importante incertitude.

Les tris croisés (origine départementale du répondant avec amélioration 
Tableau 5 : Les conséquences de la fusion sur l'image et la notoriété de l'Auvergne (Juillet 2014)

Pensez-vous que la fusion avec Rhône Alpes permettra d'améliorer:

\begin{tabular}{|l|c|c|c|c|c|c|c|c|}
\cline { 2 - 10 } \multicolumn{1}{c|}{} & \multicolumn{2}{c|}{ Oui } & \multicolumn{2}{c|}{ Non } & \multicolumn{2}{c|}{ Je ne sais pas } & \multicolumn{2}{c|}{ Total } \\
\cline { 2 - 10 } \multicolumn{1}{c|}{} & Eff & $\%$ Rep. & Eff & $\%$ Rep. & Eff & $\%$ Rep. & Eff & $\%$ Rep. \\
\hline $\begin{array}{l}\text { L'image de marque } \\
\text { de l'Auvergne }\end{array}$ & 93 & $41,5 \%$ & 84 & $37,5 \%$ & 47 & $21 \%$ & 224 & $100 \%$ \\
\hline $\begin{array}{l}\text { La notorité de } \\
\text { l'Auvergne }\end{array}$ & 99 & $44,6 \%$ & 83 & $37,4 \%$ & 40 & $18 \%$ & 222 & $100 \%$ \\
\hline Total & $\mathbf{1 9 2}$ & $\mathbf{4 3 \%}$ & $\mathbf{1 6 7}$ & $\mathbf{3 7 , 4} \%$ & $\mathbf{8 7}$ & $\mathbf{1 9 , 5 \%}$ & $\mathbf{4 4 6}$ & $\mathbf{1 0 0 \%}$ \\
\hline
\end{tabular}

de l'image de marque de l'Auvergne suite à la fusion avec Rhône-Alpes) confrontent cette image contrastée quant aux effets de la fusion : les répondants du Cantal sont très sceptiques, seulement $22,2 \%$ entrevoient des effets positifs à la différence des répondants de l'Allier plutôt enthousiastes (55\%). Les résultats pour la Haute-Loire $(47,8 \%)$ et le Puy-de-Dôme $(44,4 \%)$ se situent dans une position intermédiaire. Ces résultats sont concordants avec la perception des effets de la fusion sur l'attractivité de l'Auvergne. En effet, seuls $27,8 \%$ des répondants de l'Allier pensent que la fusion améliorera l'attractivité de l'Auvergne. On retrouve des résultats contrastés en croisant les catégories de répondants avec les effets de la fusion sur l'attractivité : les entreprises $(42,2 \%)$ et les associations $(37,5 \%)$ sont plutôt pessimistes sur les bénéfices de la fusion à la différence des collectivités $(60 \%)$ et des universités et écoles $(63,6 \%)$ qui la perçoivent globalement positivement.
L'amélioration de la notoriété et de l'image de l'Auvergne sont en partie conditionnées au choix de l'appellation de la nouvelle région au maintien du terme Auvergne dans cette appellation. Le choix d'un nom tel que Dauphiné ou Rhôdanie ôterait toute référence au territoire (géographique) auvergnat et nuirait à sa lisibilité. L'ancrage administratif de la marque ANM serait alors remis en cause alors même que cette marque et le projet de territoire (Lynch, 1960) qu'elle porte commencent à devenir visibles (tableau 6).

La grande majorité des répondants 95,2\% connaissent la marque Auvergne Nouveau Monde et $86,3 \%$ s'accordent sur la nécessité de conserver la structure porteuse de la marque auvergnate. Pour $77,4 \%$ des répondants, elle " porte une vraie ambition pour l'Auvergne ». Les éléments constitutifs des principes généraux de la plupart des marques territoire sont présents : une marque collective et 
Tableau 6: La perception de la marque ANM (Juillet 2014)

Pensez-vous que que cette marque?

\begin{tabular}{|l|c|c|c|c|c|c|c|c|}
\cline { 2 - 9 } \multicolumn{1}{l|}{} & \multicolumn{2}{c|}{ Oui } & \multicolumn{2}{c|}{ Non } & \multicolumn{2}{c|}{ Sans Opinion } & \multicolumn{2}{c|}{ Total } \\
\cline { 2 - 9 } \multicolumn{1}{l|}{} & Eff & $\%$ Rep. & Eff & $\%$ Rep. & Eff & $\%$ Rep. & Eff & $\%$ Rep. \\
\hline $\begin{array}{l}\text { Porte une vraie } \\
\text { ambition pour } \\
\text { l'Auvergne }\end{array}$ & 171 & $77,4 \%$ & 19 & $8,6 \%$ & 31 & $14 \%$ & 221 & $100 \%$ \\
\hline $\begin{array}{l}\text { Permet de transfor- } \\
\text { mer positivement } \\
\text { l'im... }\end{array}$ & 149 & $68 \%$ & 36 & $16,4 \%$ & 34 & $15,5 \%$ & 219 & $100 \%$ \\
\hline $\begin{array}{l}\text { Puisse être une } \\
\text { marque collective } \\
\text { et pa... }\end{array}$ & 145 & $66,2 \%$ & 42 & $19,2 \%$ & 32 & $14,6 \%$ & 219 & $100 \%$ \\
\hline $\begin{array}{l}\text { Porte bien les } \\
\text { valeurs de } \\
\text { l'Auvergne? }\end{array}$ & 142 & $64,5 \%$ & 38 & $17,3 \%$ & 40 & $15,2 \%$ & 220 & $100 \%$ \\
\hline $\begin{array}{l}\text { Est avant tout un } \\
\text { slogan plus qu'une } \\
\text { ma... }\end{array}$ & 117 & $53,4 \%$ & 66 & $31,1 \%$ & 36 & $15,4 \%$ & 219 & $100 \%$ \\
\hline Ne sert à rien? & 11 & $5,3 \%$ & 145 & $70 \%$ & 51 & $24,5 \%$ & 207 & $100 \%$ \\
\hline \begin{tabular}{l} 
Total \\
\hline
\end{tabular} & 192 & $\mathbf{4 3} \%$ & 167 & $\mathbf{3 7 , 4 \%}$ & $\mathbf{8 7}$ & $19,5 \%$ & $\mathbf{4 4 6}$ & $100 \%$ \\
\hline
\end{tabular}

partagée $(66,2 \%)$, portant les valeurs du territoire $(64,5 \%)$ et pouvant permettre de transformer positivement I'image du territoire (68\%). La marque ANM semble exister dans une forme affaiblie : $53,4 \%$ la considèrent plus comme un slogan lui ôtant ainsi une partie de sa substance (projet collectif partagé), de même pour près de $20 \%$, elle ne peut pas être collective et partagée.

Ces réponses mettent en évidence un questionnement ouvert sur l'usage qui est fait ou pourrait en être fait de la marque ANM.

\section{Discussion et conclusion}

Les résultats de l'enquête réalisée après l'annonce de la réforme territoriale et de la nouvelle configuration des régions françaises montrent que I'attractivité reste pour les acteurs économiques auvergnats une priorité importante et ce probablement d'autant plus dans un contexte où la région avec laquelle l'Auvergne fusionne dispose d'un pouvoir d'attraction fort. L'Auvergne souffre d'un déficit de notoriété et $d$ 'image sur lesquelles l'action de la marque ANM n'a eu qu'un effet 
relatif. Cette recherche permet de dresser trois constats majeurs :

- Le premier concerne la fragilité de la marque ANM, celle-ci, du fait de sa jeunesse, peine encore à exprimer un positionnement fort et partagé. Le positionnement choisi, "le nouveau monde », semble aller à l'encontre des éléments d'attractivité naturels de la région (la nature) mais s'inscrit plutôt bien dans l'idée de projet de territoire même s'il est perçu comme un slogan de communication.

- Le second met en évidence un effet de la promotion plus sectoriel que global si on se réfère à la progression des scores d'attractivité attestant d'une forme de spécialisation naturelle de la marque.

- Le troisième constat renvoie à la fonction même de la marque, elle constitue en effet plus un outil destiné à amener les acteurs d'un même territoire à bâtir et partager un projet qu'un outil de promotion externe.

La notoriété insuffisante, le positionnement flou, I'appellation de la marque ANM portant référence à l'Auvergne sont des éléments si on se réfère aux travaux d' Aimé-Garnier et Lai (2008) et Collange (2005) qui rendent inutilisable en l'état la marque pour la nouvelle région, à la différence de marque telle que "Sud de France » par exemple. Partant de ce diagnostic et de l'enjeu qui est de faire exister le nouveau territoire issu de la réforme, de lui donner une identité, deux pers- pectives sont envisageables : la première serait marquée par la volonté d'affirmer sa singularité, la seconde $s^{\prime}$ ancrerait dans l'amenuisement des identités des actuelles régions :

- La première perspective semble aller à l'encontre de l'esprit même de la réforme qui consiste à créer de grands ensembles pouvant exister sur la scène européenne. On peut difficilement envisager le maintien de marques dont le champ d'action concerne toutes les facettes de l'attractivité : population, entreprises, touristes, étudiants sur le périmètre administratif de l'ancienne Auvergne, et cela d'autant plus que les futures politiques régionales concerneront la totalité du territoire administré.

- La deuxième perspective, l'abandon de la marque ANM pourrait être envisagé, d'une part à la faveur d'une homogénéisation des pratiques globales de promotion du nouvel ensemble, et d'autre part car le financement de l'actuelle structure portant la marque ANM provient très majoritairement du conseil régional d'Auvergne (financée à $40 \%$ par le conseil régional)8. De plus, on peut noter une absence d'unicité dans la façon dont les répondants

\footnotetext{
8 "Le reste du budget, soit un total de 1 million d'euros, se répartit entre des fonds européens $(30 \%)$ et des fonds privés $(30 \%)$ «Plus que la réforme, ce sont les financements qui nous inquiètent ", admet Jean Pinard, directeur de la marque ANM, (Les échos, le 11/03/2015).
} 
envisagent l'existence de l'Auvergne post-fusion. $44,4 \%$ des répondants du Cantal pensent que la notion Auvergne va s'amenuiser conduisant à un réinvestissement du périmètre départemental. Pour les répondants de la Haute-Loire et du Puy-de-Dôme l'impact de la fusion sur la notion $d$ 'Auvergne est jugée minime : seuls $4,3 \%$ des répondants de la HauteLoire et $10,8 \%$ de ceux du Puy-deDôme penchent en faveur d'une disparition de la notion d'Auvergne.

Certains résultats de l'enquête soutiennent cette option. En effet, pour plus de la moitié des répondants la promotion actuelle de l'Auvergne est jugée non satisfaisante, la marque est plus perçue comme un slogan et les apports de l'adossement à RhôneAlpes en matière de notoriété et d'image sont évalués positivement. Cependant, compte tenu de la notoriété de la marque ANM auprès des acteurs socio-économiques et de l'investissement réalisé sur cet outil largent, temps consacré, mobilisation des parties prenantes, construction d'un réseau d'acteurs), il serait dommage de ne pas utiliser ce « capital». Aussi, il pourrait être opportun de redéfinir le périmètre de la marque, elle pourrait devenir un outil de promotion, par exemple exclusivement économique ou exclusivement touristique, et ainsi être recentrée sur une cible particulière, les entreprises et les porteurs de projet ou les touristes.
Le scénario (dans le cadre de la fusion Auvergne-Rhône-Alpes, le plus plausible) semble être celui de la redéfinition du périmètre d'action sectoriel de l'actuelle marque ANM globale. La marque ainsi redéfinie serait compatible avec la communication qui sera mise en place dans le nouvel ensemble et la signature institutionnelle (voire l'éventuelle marque) que la nouvelle région développera, ANM devenant en quelque sorte une marque produit de la marque institutionnelle AuvergneRhône-Alpes tout comme Only Lyon.

Les apports de la recherche se situent dans la proposition de scenarios de marquage que peut engendrer la réforme territoriale en se fondant sur les recherches réalisées sur l'identité nominale et la fusion de marques et $d^{\prime}$ entreprises et leur transposition aux marques région et aux collectivités qui les portent. Une limite provient de la difficulté à évaluer la réalité des scenarios proposés. En effet, l'enquête conduite majoritairement auprès des acteurs économiques de la région Auvergne, ne donne que des indices sur un scénario prépondérant. Pour une confirmation du scénario dominant, il serait nécessaire d'élargir l'enquête à d'autres acteurs (institutionnels, citoyens) qui ne soient pas uniquement issus du monde de l'entreprise. Une réflexion sur la redéfinition de ces " anciennes » marques région pourrait alors être engagée pour leur donner la possibilité de continuer à exister dans 
ces nouveaux ensembles, et cela d'autant plus si les acteurs politiques dans la volonté d'affirmer leur existence et de légitimer les choix politiques sont eux-mêmes conduits à développer leur propre marque.

\section{Bibliographie}

AAKER D. A., KELLER, K. L., 1990, "Consumer evaluations of brand extensions", The Journal of Marketing, pp. 27-41.

AIME-GARNIER I., LAI C., 2008, " Le changement de nom de marque: définition, clarification et proposition d'une typologie ", Décisions Marketing, pp. 33-46.

AITKEN R., CAMPELO A., 2011, "The four Rs of place branding", Journal of Marketing Management, 27(9-10), pp. 913-933.

ANHOLT S., 2006, "Is place branding a capitalist tool?", Place Branding, 2(1), pp. 1-4.

BANERJEE S., 2008, "Logo Change: Little Image Touch up or Serious Overhaul! Imperatives and Scanning Parameters", Journal of Marketing \& Communication, May-August, Vol. 4, Issue 1, pp. 59-70.

BANKS S.P., 1990, "Organizational Power as Communicative Praxis", in Corman S.R., Banks C.R., Bantz S.P., M.E. Mayer (Eds), Foundations of Organizational Communication, New-York: Longman, pp. 44-61.

BOISTEL P., 2012, «Comment qualifier le message lors d'un changement de nom d'entreprise ? Analyse de quatre cas d'entreprises ", Gestion 2000, 3, Volume 29, pp. 97-113.

CARDY H., 1997, «Territoires incertains et Communication publique», QUADERNI, $\mathrm{n}^{\circ} 34$, hiver, pp. 111-127.
CEGARRA J. J., MICHEL G., 2001, "Co-branding: clarification du concept », Recherche et applications en marketing, 16(4), pp. 57-69.

CEGARRA J. J., 1994, "Gérer un portefeuille de marques », Décisions Marketing, pp. 81-91.

CHANUT V., ROCHETTE C., 2012, La fabrique d'une marque Région: le cas de la marque «Auvergne Nouveau Monde». Politiques et management public, 29(3), pp. 493-517.

COLLANGE V., 2005, " L'impact du changement de nom de marque sur l'attitude des consommateurs ", Doctoral dissertation, Thèse de Doctorat en Sciences de Gestion, Université Paul Cezanne, Aix-Marseille III, Institut d'Administration des Entreprises.

DOWLING G. R., 1996, "Corporate identity traps", Working paper-australian graduate school of management.

HOUILLIER-GUIBERT C.-E. (2012), « De la communication publique vers le marketing des territoires: approche microsociologique de la fabrication de l'image de marque ", Gestion et management public, vol. 1, n², pp. 35-49.

KAPFERER J.-N., 1988, "Maîtriser l'image de l'entreprise : le prisme d'identité », Revue Française de Gestion, 71, pp. 76-82.

KAPFERER J. N., 1991, « Les marques, capital de l'entreprise », (p. NC). Editions d'Organisation.

KAPFERER J.-N., 1994, "Strategic Brand Management: New Approaches to Creating and Evaluating Brand Equity", Simon and Schuster Eds.

KAPFERER J. N., 2002, "Corporate brand and organizational identity", Corporate and organizational identities: Integrating strategy, marketing, communication and organizational perspectives, pp. 175-194.

KELLER K.L., 2002, "Branding and brand eqvity", Handbook of marketing.

KLINK R. R., 2003, "Creating meaningful brands: The relationship between brand name and brand mark", Marketing Letters, 14(3), pp. 143-157. 
LAURENT B., 2006, « Nom de marque, nom de produit. Production de sens en discours publicitaire », Thèse de doctorat. Montpellier 3.

LEJEUNE C., VAS A., 2011, "Identité, changement et proximité organisationnelle », Revue française de gestion, (4), pp. 171-187.

LIPIANSKY E. M., 1993, "L'identité dans la communication", Communication et langages, 97(1), pp. 31-37.

LYNCH K., 1960, "The image of the city", (Vol. 11). MIT press.

MERRILEES B., MILLER D., 2008, "Principles of corporate rebranding", european Journal of Marketing, 42(5/6), pp. 537-552.

MICHEL G., 2004, Au cœur de la marque. Les clés du Management de la marque.

MOILANEN T., RAINISTO S., 2008, "How to brand nations, cities and destinations: a planning book for place branding", Palgrave Macmillan.

MUZELLEC L., LAMBKIN M., 2006, "Corporate rebranding: destroying, transferring or creating brand equity?" European Journal of Marketing, 40(7/8), pp. 803-824.
MUZELLEC L., 2006, "What is in a Name Change? Re-Joycing Corporate Names to Create Corporate Brands", Corporate Reputation Review, Vol 8, n4, pp. 305-321.

RIGAUD E., 2009, "Fusion terminée, fusion interminable ? Le cas Peugeot-Citroën », Gérer et Comprendre. Annales des Mines, 1, n ${ }^{\circ}$ 95, pp. 26-34.

ROCHETTE C., 2012, "L'approche ressources et compétences comme clé de lecture du processus d'élaboration d'une ressource originale : la marque territoire ", Gestion et management public, Volume $1 / n^{\circ} 1, n^{\circ} 1$, pp. 4-20.

VALETTE-FLORENCE R., DE BARNIER V., 2011, "Evaluation de la marque presse par son lecteur: Place et apport de la personnalité de la marque ». Gestion 2000, Vol. 28, (n 5,), pp. 62-83.

ROY D.F., 1960, "Banana Time: Job satisfaction and informal interaction", People and Organizations, 18, pp. 158-168.

STUART H., MUZELLEC L., 2004, "Corporate makeovers: Can a hyena be rebranded?", The Journal of Brand Management, $11(6)$, pp. 472482. 